such a handicap to the Colonial scientific worker in the past. If it should prove, furthermore, that the report of the visiting mission to the Cameroons betokens a wider appreciation of the work of the whole Colonial Service, there is every reason to hope that, with the new conditions of service, the Colonial Office will no longer find itself unable to obtain sufficient recruits of the high quality desired for its Research Service or for any other branch.

${ }^{1}$ Colonial Office. Appointments in His Majesty's Colonial Service. Pp. 111. (London: H.M. Stationery Office, 1950.) 1s. net

${ }^{3}$ Colonial Office. Appointments in His Majesty's Colonial Research Service. Pp. 15. (London: Colonial Office. 1950.)

\section{ADVANCES IN ENZYMOLOGY}

\section{Advances in Enzymology and Related Subjects of} Biochemistry

Edited by F. F. Nord. Vol. 8. Pp. xi+538. 48s. Vol. 9. Pp. $\mathrm{x}+760$. 54s. (New York and London : Interscience Publishers, Inc., 1948 and 1949.)

T HESE two volumes contain twenty-two articles on a variety of subjects of enzymological and biochemical interest. They vary from a study of the function of cytoplasm to a discussion on industrial biosynthesis. They consist of authoritative essays, some of them extensive monographs, in special fields of biochemistry, and they well uphold the high standard set in previous volumes of "Advances in Enzymology".

Dr. Ludwik Monné gives a critical review of recent literature dealing with the structure of protoplasm, and he attempts to portray a consistent picture of the functioning of eytoplasm. He describes investigations on chromidia and shows how new work has demonstrated that ribonucleic acid is present within the cytoplasm (in the chromidia) and that thymonucleic acid is in the chromosomes. Chromidia and mitochondria differ from each other, the latter lacking ribonucleic acid. Nucleic acids appear to be concerned with mechanisms of protein synthesis, and it is claimed that "self-perpetuating corpuscles must be nucleoproteins". The chromidia are an organised system of chemical compounds and are self-perpetuating bodies similar to chromatín. Dr. Monné proceeds to compare various living fibrils and discusses plasma membranes, mitochondria, Golgi bodies, chloroplasts and cell organisation. An interesting and stimulating discussion of the metabolic activities of the cell follows, with comments on contractility, mobility, irritability, conductivity and permeability.

Drs. M. A. Lauffer, W. C. Price and A. W. Petre write on the nature of viruses, dealing with the plant and bacterial varieties. Virus mutations are discussed, and it is pointed out that studies on inheritance in viruses have shown that these entities are more highly organised than genes. They contain, in fact, a number of genes or gene-like structures. Great progress has been made in the last decade on the elucidation of the nature of viruses, the impulse being given by the crystallization and characterization of tobaceo mosaic virus. Later work showing that this is a nucleoprotein has resulted in the application of the new techniques to purification of many other viruses. Details are given of their physical constants, and shadow electron micrographs of virus particles are included. All the plant viruses, as well as some of the animal viruses, seem to consist of nucleoproteins ; others, like vaccinia virus, also contain fat and carbohydrate. Tobacco mosaic virus consists of sixteen to eighteen amino-acids, the number depending upon the strain.

A most informative article on the chemistry and enzymology of nucleic acids is contributed by Dr. F. Schlenk. This is particularly useful in view of the paucity of comprehensive reviews and monographs on the subject. The compounds discussed are classified into two main groups : one comprising ribo- and desoxyribo-nucleic acids and their split products; and the other comprising co-enzyme nucleotides such as adenine, riboflavin and nicotinamide nucleotides. The nucleotides are defined as the phosphoric acid esters of the nucleosides. The latter are the $\mathrm{N}$-glycosides of naturally occurring bases, the carbohydrate being $d$-ribose, $d$-2-desoxyribose or some closely related sugar. The nucleic acids or polynucleotides are not yet clearly defined, and it is preferable at present to use the non-committal terms of ribonucleic acid and desoxyribonucleic acid, these being understood as collective terms, since evidence is appearing that each type is collective in nature. Dr. Schlenk discusses aspects of the metabolism of the constituents of nucleic acids, and describes recent spectrophotometric studies of these substances, pointing out the value of the application of spectrophotometry to enzyme and cytoplasmic studies. The enzymes hydrolysing the nucleosides, nucleotides and nucleic acids receive considerable attention, and a most interesting discussion is given of the architecture of the nucleic acids. It is emphasized that a new development in the nucleic acid field is the tendency to question the existence of distinct tetranucleotides as structural units of nucleic acids. In spite, however, of the increased interest in the chemistry and enzymology of nucleic acid, research activity in this field needs intensification. It is possible that this may take place now that welldefined substrates (nucleosides, nucleotides and nucleic acids) are becoming available for enzyme studies.

One of the most important and interesting articles in the series under review is that by Dr. T. Mann on the metabolism of semen. This subject, to which Dr. Mann has, himself, contributed much of great value, has become of considerable significance, and doubtless will achieve greater importance as time goes on. Dr. Mann points out that although it is true that the best way to prolong the life-span of sperm cells is to reduce their motility and metabolism, say, by lowering the temperature, the mammalian spermatozoa are to a large extent independent of the small nutrient reserve they possess. They obtain their energy by assimilation and metabolism of fructose present in the seminal plasma. So far as nutrition is concerned, the spermatozoa "resemble more a culture of microorganisms in a nutrient medium rather than the other animal tissues with their complicated supply of nutrient material via the blood capillaries". Another characteristic of the sperm cell is its remarkably high permeability, which accounts for the high velocity of the exchange reactions taking place between the sperm cells and surrounding medium. Moreover, large molecules, for example, enzymes such as hyaluronidase, can detach themselves from the sperm structure and are released into the outer medium. Perhaps such phenomena are involved in the fertilizing function of the spermatozoa. A detailed account is given of the constituents making up semen, with particular reference to sperm nucleic acid and adenosinetriphosphate and to such enzymes as 
hyaluronidase and phosphatases. Fructose and fructolysis in sperm metabolism receive close atten. tion. There seems little doubt that oxidation of carbohydrate predominates over other aerobic processes in these cells, constituting their major source of metabolic energy. A variety of substances other than carbohydrate may, however, be oxidized by the spermatozoa, and it has been claimed. for example, that acotic acid may be a source of oxidation energy for bull spermatozoa. There is very little catalase present in normal bull semen, which may account for the toxicity of hydrogen peroxide to spermatozoa and possibly also for the harmful effect of pure oxygen on sperm motility. There is still insufficient knowledge to appraise properly the relationships between fertilizing capacity and metabolism of spermatozoa; but much progress in this important biological problem is now being made.

"Mechanism of Fertilisation in Metazna" is the subject of an extensive and authoritative article by Dr. J. Runnström. This deals with the dependence of fertilizability on the physiological state of the sex cells, and on the interacting substances produced by the germ cells. The structural and metabolic changes following fertilization and artificial parthenogenesis are described in detail. Enzyme activation as a major factor in the incitement of development is discussed. Dr. Runnström has recently emphasized the view that development may be dependent on the activation of enzymes, which are controlled in the cell by inhibitors and activators. There seems little doubt that fertilization and enzyme activity are closely connected. For example, Orström contends that fertilized eggs can form glutamine from added ammonia and glutamic acid, whereas unfertilized eggs are unable to accomplish this synthesis but can break down glutamine to glutamic acid and ammonia. Release of calcium, which may occur on fertilization of the eggs, seems to play a decisive part in enzyme activation.

Fat metabolism receives considerable attention in Vol. 8, in articles on the synthesis of lipides by A. Kleinzeller, on the biochemistry of fatty acid cata. bolism by F. L. Breusch, and on lipoxidase and the auto-oxidation of unsaturated fatty acids by $S$. Bergström and R. T. Holman. An article on the present status of the "Antifatty-Liver Factor of the Pancreas" by I. L. Chaikoff and C. Entenman may be included in this group. These articles give a clear over-all picture of the state of knowledge on fat metabolism as seen a year or two agn. Dr. Breusch's article on fatty acid oxidations, written in memory of Franz Knoop who carried out the classical work in this field, is particularly welcome. It represents a detailed account of recent work showing the integration of fatty acid and carbohydrate metabolism. Dr. Breusch's own work in this field is described, and his views are put forward with clarity. He shows how recent work gives rise to the conclusion that the greater part of the catabolism of fatty acids in warmblooded animals is brought about by a gradual interaction of the muscles and kidneys on one hand, and hy the liver on the other. It is accomplished in such $a$ way that liver produces acetoacetic acid and $\beta$-hydrnxybutyric acid from fatty acids by multiple alternate $\beta$-oxidation, by way of two-carbon fragments. These acids are then completely oxidized in the muscles and kidneys by way of the $\mathrm{C}_{B}$-tricarboxylic cycle. Dr. Kleinzeller summarizes recent work on fat synthesis, describing the conditions necessary for fat formation and the various substances from which fat may arise. He discusses mechanisms of fatty acid synthesis and comments on the work that has been carried out on the synthesis of phospholipides, sterols and waxes. He emphasizes the role of the two carbon fragment in fat synthesis, a process which is becoming increasingly important as work with isotopic carbon as a tracer progresses. Drs. Bergström and Holman deal with the phenomena of the autoxidation of unsaturated fats, a matter of considerable practical as well as biological importance. The antioxidants, and the possible role of tocopherol in this connexion, are discussed. The new conception in nutrition that fatty livers may arise from a deficiency of labile methyl groups in the diet is the subject of study in an interesting paper by Drs. Chaikoff and Entenman. They go fully into the problem of the interrelationships between choline, methionine and the factors present in the pancreas that affect fat formation in the liver.

Snake venoms are the subject of an article by Dr. E. A. Zeller. He describes the presence in venom of a variety of hydrolytic enzymes, including choline esterase and hyaluronidase, and he points out that $l$-amino-acid oxidase (which differs from the mammalian variety) is the only non-hydrolysing enzyme so far found in snake venoms. Many lesions of brain and nerve are caused by snake venoms, the attack of the neurotoxins being directed towards different parts of the nervous system. There seem to be good reasons for believing that enzyme processes are involved in the action of the neurotoxins ; but there is little exact information, and no clear relationships are yet established.

Dr. R. F. Dawson writes a valuable account of the present knowledge of alkaloid biogenesis. He deals with the origin, development and accumulation of alkaloids such as nicotine, nor-nicotine, anabasine, hyoscyamine, hyoscine and the cinchona group. Mechanisms of synthesis are discussed, and there is an interesting section on the relations between alkaloid synthesis, protein metabolism and plant physiology.

The dehydropeptidases receive expert treatment at the hands of Dr. J. P. Greenstein. These enzymes act specifically upon peptides containing an $\alpha \cdot \beta$ double bond adjacent, to the substituted amide bond, and are distinct from the peptidases such as dipeptidase, carboxy peptidase, etc., that catalyse the hydrolysis of the peptide bond uniting the normal saturated $\alpha$-amino-acids. The dehydropeptides promise to be of considerable importance in metabolic processes, and Dr. Greenstein's article is most timely.

Limitations of space alone forbid more than mention of the very valuable articles by Dr. M. Heidelberger and Dr. M. Maver on quantitative studies on complement; Dr. F. F. Nord and Dr. J. C. Vitucei on the microbiological degradation of cellulose ; Dr. H. E. Street on nitrogen metabolism of higher plants; Dr. A. D. MeLaren on the effects of light and radiation on enzymes, hormones and viruses; Dr. A. E. Stearn on the kinetics of biological, especially enzymic, reactions; the late Dr. L. Michaelis on reversible step reactions; Dr. D. Glick on the principles of cytochemistry; Dr. T. K. Walker on acid formation in fungal metabolism; Dr. M. A. Joslyn on enzyme activities in refrigerated vegetable tissues; and by Dr. A. Hesse on the industrial biosynthesis of fat. These articles are all of the highest standard, and no research worker in the biochemical or enzymological field can afford to neglect them.
J. H. QuAsTEL 